\title{
Study of the in vitro cytotoxicity testing of medical devices (Review)
}

\author{
WEIJIA LI, JING ZHOU and YUYIN XU \\ Henan Province Institute for Medical Equipment Testing, Zhengzhou, Henan 450003, P.R. China
}

Received May 12, 2015; Accepted May 28, 2015

DOI: $10.3892 /$ br.2015.481

\begin{abstract}
The cytotoxicity test is one of the biological evaluation and screening tests that use tissue cells in vitro to observe the cell growth, reproduction and morphological effects by medical devices. Cytotoxicity is preferred as a pilot project test and an important indicator for toxicity evaluation of medical devices as it is simple, fast, has a high sensitivity and can save animals from toxicity. Three types of cytotoxicity test are stated in the International Organization for Standardization 109993-5: Extract, direct contact and indirect contact tests. The xCELLigence real-time cell analysis system shows a significant potential in regards to cytotoxicity in recent years. The present review provides a brief insight into the in vitro cytotoxicity testing of medical devices.
\end{abstract}

\section{Contents}

1. Introduction

2. Detection methods

3. Outlook

\section{Introduction}

Biocompatibility is concerned with medical devices in a specific environment and location of the host, their ability to react with each other when directly or indirectly in contact with the host, the biological properties of medical devices when static, the dynamic process of change in vivo and the ability to tolerate all the host systems while maintaining relative stability, with no exclusion and destruction (1-3). Medical devices must undergo rigorous testing to determine their biocompatibility when they have contact with the body, regardless of their mechanical, physical and chemical properties or how good

Correspondence to: Ms. Yuyin Xu, Henan Province Institute for Medical Equipment Testing, 5 Jingyi Road, Zhengzhou, Henan 450003, P.R. China

E-mail: 2363401540@qq.com

Key words: cytotoxicity, biocompatibility, medical devices, xCELLigence they are until they are used in the human body, according to the International Organization for Standardization (ISO) and national standards $(4,5)$. With the continuous development of science and technology, a new medical device must undergo biocompatibility tests of cytotoxicity, sensitization, intradermal irritation, acute systemic toxicity and a series of tests prior to entering a clinical environment to ensure safe and effective use for humans.

Medical devices have been widely used in various clinical disciplines (6). As these devices have direct contact with the tissues and cells of the body, they not only require good physical and chemical properties, but must also have good biocompatibility (7). Security for medical devices has increased in value by governments. A series of international standards for medical devices (ISO 10993) have been published by ISO in 1992, and scientists in China began studying methods for evaluation of medical devices since the late 1970s, thus ensuring safety in the research, production and clinical use, and promotion of the development of medical devices $(8,9)$. Among the biocompatibility tests, cytotoxicity is preferred as a pilot project test and as an important indicator for the evaluation of medical devices as it is simple, fast, has a high sensitivity and can save animals from toxicity $(10,11)$.

The cytotoxicity test, one of the biological evaluation and screening tests, uses tissue cells in vitro to observe the cell growth, reproduction and morphological effects by the medical devices (12). Cytotoxicity is one of the most important methods for biological evaluation as it has a series of advantages, along with the preferred and mandatory items (11). Part 5 of the biological evaluation of medical devices in ISO 10993-5 is as follows: Regulations of cytotoxicity in vitro, countries have to make the relevant provisions of the corresponding cytotoxicity tests according to their actual situation (13). With the continuous development of cytotoxicity tests, methods, such as detection of cell damage by morphological changes, determination of cell damage, measuring cell growth and metabolic properties, have appeared and have gradually been developed from qualitative evaluation to quantitative (14-17). However, the correlation remains to be further studied, as well as the evaluation of the correlation results of these methods with other biological evaluation. Due to the diversity of medical devices, the variability of the environment of the body and the complexity of the interaction between the body and medical devices, a uniform evaluation method or cytotoxicity test evaluation system has not been established thus far. 
Three types of cytotoxicity test are stated in ISO 10993-5: Extract, direct contact and indirect contact tests (including agar overlay assay and filter diffusion). In general, the extract test is suitable for detecting the toxicity of soluble substances of medical devices and is usually consistent with the results of animal toxicity tests. The direct contact assay is the most sensitive for testing the cytotoxicity of the medical devices; the medical devices can be measured even with weak cytotoxicity (18). The agar overlay assay is suitable for the medical devices that have large toxicity and bulk filtering (19) and the molecular filtration method is suitable for the biocompatibility evaluation of the toxic components of small molecular weight medical devices (20). Gao et al (21) identified a good correlation between the direct and indirect contact test and a lesser association between the extract test and the other two trials.

\section{Detection methods}

Extract test. The mitochondrial dehydrogenase performance measurement, also known as the 3-(4,5-dimethyl-2-thiazolyl)2,5-diphenyl-2H-tetrazolium bromide (methyl thiazolyl tetrazolium; MTT) assay, is a rapid assessment of cell proliferation and cytotoxicity colorimetric assay to measure cell metabolism or function used (22). The main principle is as follows: Mitochondrial dehydrogenase in the cytochrome $b$ and $c$ sites of the living cells can cleave the tetrazole ring, and the yellow, water-soluble MTT is reduced to produce a purple crystalline formazan. This substance is soluble in dimethyl sulfoxide and other organic solvents, but is insoluble in water. The amount of crystals formed has a positive correlation to the number of cells and their activity, and measuring the absorbance (optical density) colorimetric value reflects the number of surviving cells and metabolic activity.

The MTT assay is currently the most commonly used method to test cell growth rate and toxicity of the culture. Although the MTT assay has a sensitive response to the proliferation of medical devices, it has numerous problems in application. For example, the test results were not the same when using different doses of extract from natural latex rubber condoms. A previous study reported on 15 brands from 6 batches of condoms $\left(6 \mathrm{~cm}^{2} / \mathrm{ml}\right.$ proportion extraction) assessed by the MTT assay. When the extract doses were 50 , 20 and $10 \%$, the cell toxicity grades were 4, 2 and 1, respectively. However, when these medical devices were detected by the indirect method, all had a cell toxicity of grade 2 . Similar results occurred with assessment of disposable catheters and medical gloves: When the doses of the extract tested were $50-100 \%$, all the cytotoxicity results were grade 4 ; however, when the dose was $25 \%$, the test results were distributed in grades 4, 3 and 2 (23). Cytotoxicity of an ultrasound-coupling agent showed that the same method of extraction leads to different results as the cell densities were not the same; this was not the case with the indirect method. Certain investigators have studied the effects of concentration and time on cell cytotoxicity and found that when the cell concentration was not the same, the time that toxicity appears was not the same. Within a certain range, toxicity increases with time gradually (24). Thus, determining the appropriate time to evaluate the toxic effect is also essential. However, the MTT assay can easily reflect the dose-related toxicity, but requires a significant amount of time to determine the influence of time on the testing of the medical devices. In summary, although the MTT assay is more accurate than other detection methods, it is relatively simple, but it is also cumbersome. There are numerous steps in the course of the MTT assay, and it is time-consuming, repetitive and has a slightly poor outcome. Traditional cytotoxicity tests use artificial methods, such as measuring platelets to count the number of surviving cells, which can be affected by humans and environmental factors, leading to errors and a long test cycle.

\section{Test by indirect contact}

Molecular filtration. Molecular filtration detects cytotoxicity by evaluating the activity of the monolayer succinate dehydrogenase effect by the medical devices. Monolayer cells are cultured on a cellulose ester filter first and the original culture medium is subsequently replaced with medium containing agar, allowing fresh medium gel on cells. Finally, the single-cell membrane gel is separated and reversed to expose the membrane upwards. Following exposure to the sample, the filter is removed and the metabolic activity of cells affected by the sample is measured (25). This method can observe the primary and secondary cytotoxicity of medical devices, and is simple, rapid, sensitive, reliable, easy to promote and suitable for the evaluation of the short-term and mildly toxic medical devices, but it has the shortcomings of impact force from the medical devices deposited in the diffusion of the product.

Agar overlay assay. This method is used to evaluate the biological medical devices that can leach toxic substances, and it can be performed on medical devices or extracts. Prior to the addition of $1 \%$ live cells containing dye (such as neutral red) agar or agars (low melting point) to update the trial media, the monolayer should be cultured first. Agar placed between the cells and the medical devices on the agar forms a barrier. Nutrients, gases and soluble toxic substances can penetrate and diffuse the agar (26). This is a semi-quantitative test method, and the degree of destruction of the membrane of cells are estimated by electron microscopy or the radius of the dissolution and bleaching zones is visualized. This method is simple, rapid, inexpensive and easy to promote. As there is an agar isolation layer between the medical devices and the cell, this method is suitable for screening a wide range of medical devices and large quantities of toxic medical devices. Motsoane et al (27) used this method to evaluate 11 types of biological medical devices, and the results were satisfactory and suggest that the key to ensure the success of this experiment is the proper use of neutral red dye and controlled temperature of agar medium. However, the presence of agar cannot adequately represent the barrier in vivo, and sensitivity is also vulnerable and can be affected by the extent of leachables that can diffuse in the agar. This method has high sensitivity for small molecular weight and water-soluble extractables (low sensitivity and vice versa), There is, however, susceptibility to subjective factors in the results (area of fade and dissolve proportions).

Direct contact method. The direct contact method yields direct contact of the solid medical devices with cultured mammalian cells in vitro. The cytotoxic test occurs by observing the morphological changes and detecting the changes in the number of cells; it can directly reflect the impact of testing the 
medical devices on the cells. Although the method has high sensitivity, it is more demanding for the medical devices, and suitable medical devices are limited (28). For example, certain less dense medical devices, such as condoms, float easily in the medium, and as the cells are difficult to access, this law does not apply for testing (29).

Other methods. Cell growth inhibition tests, the ultraviolet spectrophotometer assay, cell rehabilitation method, the degree of cell proliferation assays, cell morphology observation, dentin barrier and high-pressure liquid chromatography are used for the cytotoxicity analysis. In recent years, the evaluation methods have been developed from the whole animal and cellular level to the molecular level using molecular biology techniques, such as the activation of proto-oncogenes and tumor-suppressor gene inactivation studies. Investigators have reported the restoration of the precipitated metal ions on the oral mucosa cells and osteoblast-like cells, DNA damage and induction of apoptosis at the molecular level $(30,31)$. Markey et al (32) reported an estrogen compound (bisphenol propane) leaking from dental medical devices, and plastic products can cause changes in DNA synthesis and induce abnormal body morphology, function and behavior. Therefore, more research is required to evaluate the biological properties of dental medical devices, including potential estrogenic activity.

\section{Outlook}

The cytotoxicity test is one of the most important indicators of the biological evaluation system in vitro, and with the progress of modern cell biology, experimental methods to evaluate cytotoxicity are also continuously being developed and improved. However, there are no uniform cytotoxicity test methods, and all these existing methods have particular problems. The xCELLigence real-time cell analysis system (RTCA) is an impedance detection sensor system, and through a special process, the microelectronic sensor chip integrated into the bottom of the cell detection plate allows for a real-time, dynamic, quantitative assessment of tracking changes in cell morphology, cell proliferation and differentiation. When the electrode interface impedance changes due to cells grown on the microelectrode surface, this change shows a correlation with the real-time state, and relevant biological information can be obtained via the real-time dynamic electrode impedance detection, including cell growth, morphology and death (33). Urcan et al (34) used RTCA technology to assay the proliferative capacity of human gingival fibroblasts to investigate cytotoxicity of the most common monomers/comonomers in dental resin composites, showing that the technology has a good prospect. We are trying to introduce RTCA technology into cytotoxicity tests of medical devices in vitro, to establish a more objective and accurate method for evaluating the cytotoxicity of medical devices in vitro.

\section{Acknowledgements}

The present study was supported by the Youth Development Research Foundation of National Institutes for Food and Drug Control (grant no. 2013WC1).

\section{References}

1. Anderson JM and Shive MS: Biodegradation and biocompatibility of PLA and PLGA microspheres. Adv Drug Deliv Rev 64: 72-82, 2012.

2. Shim M, Shi Kam NW, Chen RJ,Li Y and Dai H: Functionalization of carbon nanotubes for biocompatibility and biomolecular recognition. Nano Lett 2: 285-288, 2002.

3. Shukla R, Bansal V, Chaudhary M, Basu A, Bhonde RR and Sastry M: Biocompatibility of gold nanoparticles and their endocytotic fate inside the cellular compartment: A microscopic overview. Langmuir 21: 10644-10654, 2005.

4. Black J (ed): Biological Performance of Materials: Fundamentals of Biocompatibility. 4th edition. CRC Press, Boca Raton, FL, p520, 2006 .

5. Gundarov VP and Kavalerov GI: Development of standardization and certification of the quality of medical devices. Med Tekh 35: 4-8, 2001 (In Russian).

6. Rai R, Keshavarz T, Roether JA, Boccaccini AR and Roy I: Medium chain length polyhydroxyalkanoates, promising new biomedical medical devices for the future. Medical devices Science and Engineering: R. Reports 72: 29-47, 2011.

7. Reddy LH, Arias JL, Nicolas J and Couvreur P: Magnetic nanoparticles: Design and characterization, toxicity and biocompatibility, pharmaceutical and biomedical applications. Chem Rev 112: 5818-5878, 2012.

8. Sinn S, Scheuermann T, Deichelbohrer S, Ziemer G and Wendel HP: A novel in vitro model for preclinical testing of the hemocompatibility of intravascular stents according to ISO 10993-4. J Mater Sci Mater Med 22: 1521-1528, 2011.

9. Zhang D, Zhang BP, Li ZG, Wang L, Liu B and Wang JQ: Biocompatibility of $\mathrm{HAP} / \mathrm{Ti}$ gradient coating by microarc oxidation and biomimetic process. Advanced Materials Research 177: 325-328, 2011.

10. He Q and Shi J: Mesoporous silica nanoparticle based nano drug delivery systems: Synthesis, controlled drug release and delivery, pharmacokinetics and biocompatibility. J Materials Chemistry 21: 5845-5855, 2011.

11. Kunzmann A, Andersson B, Thurnherr T, Krug H, Scheynius A and Fadeel B: Toxicology of engineered nanomedical devices: Focus on biocompatibility, biodistribution and biodegradation. Biochim Biophys Acta 1810: 361-373, 2011.

12. Soenen SJ, Manshian B, Montenegro JM, Amin F, Meermann B, Thiron T, Cornelissen M, Vanhaecke F, Doak S, Parak WJ, et al: Cytotoxic effects of gold nanoparticles: A multiparametric study. ACS Nano 6: 5767-5783, 2012.

13. Osthues RM, da Silva SN, Zavaglia CA and Fialho SL: Study of the release potential of the antibiotic gentamicin from microspheres of BCP. Key Engineering Materials 493: 269-274, 2012.

14. Uboldi C, Giudetti G, Broggi F, Gilliland D, Ponti J and Rossi F: Amorphous silica nanoparticles do not induce cytotoxicity, cell transformation or genotoxicity in Balb/3T3 mouse fibroblasts. Mutat Res 745: 11-20, 2012.

15. Piao MJ, Kang KA, Lee IK, Kim HS, Kim S, Choi JY, Choi J and Hyun JW: Silver nanoparticles induce oxidative cell damage in human liver cells through inhibition of reduced glutathione and induction of mitochondria-involved apoptosis. Toxicol Lett 201: 92-100, 2011.

16. Damas BA, Wheater MA, Bringas JS and Hoen MM: Cytotoxicity comparison of mineral trioxide aggregates and EndoSequence bioceramic root repair materials. J Endod 37: 372-375, 2011.

17. Kasper J, Hermanns MI, Bantz C, Maskos M, Stauber R, Pohl C, Unger RE and Kirkpatrick JC: Inflammatory and cytotoxic responses of an alveolar-capillary coculture model to silica nanoparticles: Comparison with conventional monocultures. Part Fibre Toxicol 8: 6, 2011.

18. De Melo WM, Maximiano WMA, Antunes AA, Beloti MM, Rosa AL and de Oliveira PT: Cytotoxicity testing of methyl and ethyl 2-cyanoacrylate using direct contact assay on osteoblast cell cultures. J Oral Maxillofac Surg 71: 35-41, 2013.

19. Sjögren G, Sletten G and Dahl JE: Cytotoxicity of dental alloys, metals, and ceramics assessed by millipore filter, agar overlay, and MTT tests. J Prosthet Dent 84: 229-236, 2000.

20. Jin CY, Zhu BS, Wang XF and Lu QH: Cytotoxicity of titanium dioxide nanoparticles in mouse fibroblast cells. Chem Res Toxicol 21: 1871-1877, 2008.

21. Gao J, Wang S, Jin M and Yan X: In vitro cytotoxicity test of medical ultrasonic couplant. Zhongguo Yi Liao Qi Xie Za Zhi 37: 210-212, 2013 (In Chinese). 
22. Fotakis G and Timbrell JA: In vitro cytotoxicity assays: Comparison of $\mathrm{LDH}$, neutral red, MTT and protein assay in hepatoma cell lines following exposure to cadmium chloride. Toxicol Lett 160: 171-177, 2006.

23. Jorge JH, Giampaolo ET, Vergani CE, Machado AL, Pavarina AC and Carlos IZ: Cytotoxicity of denture base resins: Effect of water bath and microwave postpolymerization heat treatments. Int J Prosthodont 17: 340-344, 2004

24. Sun J, Xue M and Zhang CX: Molecular filtration method to detect Cytotoxic of 13 kinds of medical polymer medical devices. Zhongguo Sheng Wu Yi Xue Gong Cheng Xue Bao 8: 60, 1989 (In Chinese).

25. Sayes CM, Liang F, Hudson JL, Mendez J, Guo W, Beach JM, Moore VC, Doyle CD, West JL, Billups WE, et al: Functionalization density dependence of single-walled carbon nanotubes cytotoxicity in vitro. Toxicol Lett 161: 135-142, 2006.

26. Oldham RK, Siwarski D, McCoy JL, Plata EJ and Herberman RB: Evaluation of a cell-mediated cytotoxicity assay utilizing 125 iododeoxyuridine-labeled tissue-culture target cells. Natl Cancer Inst Monogr 37: 49-58, 1973.

27. Motsoane NA, Bester MJ, Pretorius E and Becker PJ: An in vitro study of biological safety of condoms and their additives. Hum Exp Toxicol 22: 659-664, 2003.

28. Frei K, Siepl C, Groscurth P, Bodmer S, Schwerdel C and Fontana A: Antigen presentation and tumor cytotoxicity by interferon- $\gamma$-treated microglial cells. Eur J Immunol 17: 1271-1278, 1987.
29. Wang SQ, Chen QY, Zhang LH and Ma J: Vitro Cytotoxicity Test of Natural Latex Rubber Condom. J Prev Med Inf 28: 197-200, 2012 (In Chinese).

30. Faccioni F, Franceschetti P, Cerpelloni M and Fracasso ME: In vivo study on metal release from fixed orthodontic appliances and DNA damage in oral mucosa cells. Am J Orthod Dentofacial Orthop 124: 687-694, 2003.

31. Cortizo AM and Etcheverry SB: Vanadium derivatives act as growth factor - mimetic compounds upon differentiation and proliferation of osteoblast-like UMR106 cells. Mol Cell Biochem 145: 97-102, 1995.

32. Markey CM, Wadia PR, Rubin BS, Sonnenschein C and Soto AM: Long-term effects of fetal exposure to low doses of the xenoestrogen bisphenol-A in the female mouse genital tract. Biol Reprod 72: 1344-1351, 2005.

33. Ke N, Wang X, Xu X and Abassi YA: The xCELLigence system for real-time and label-free monitoring of cell viability. Methods Mol Biol 740: 33-43, 2011.

34. Urcan E, Haertel U, Styllou M, Hickel R, Scherthan H and Reichl FX. Real-time xCELLigence impedance analysis of the cytotoxicity of dental composite components on human gingival fibroblasts. Dent Mater 26: 51-58, 2010. 